\title{
CONDICIÓN DE SALUD DE PACIENTES DIABÉTICOS Y SU SATISFACCIÓN CON EL TRATAMIENTO PARA LA ENFERMEDAD ${ }^{1}$
}

\author{
HEALTH CONDITION OF TYPE 2 DIABETIC PATIENTS AND THEIR \\ SATISFACTION REGARDING DISEASE TREATMENT
}

\author{
María Alejandra Galiano G. * \\ María Silvia Calvo A. ${ }^{* *}$ \\ María Alicia Feito T. ${ }^{* * *}$ \\ María Waleska Aliaga B. ${ }^{* * *}$ \\ Sara Leiva M. \\ BeATriz Mujica P. ${ }^{* * * * *}$
}

\begin{abstract}
RESUMEN
La diabetes mellitus (DM) va en aumento en nuestro país, con alta prevalencia de complicaciones especialmente por mal control metabólico. Objetivo: evaluar asociación de condición de salud, variables sociodemográficas y años de diagnóstico con satisfacción del paciente con características del tratamiento de diabetes. Material y método: estudio de prevalencia en 340 pacientes de 1.100 usuarios DM2 controlados en Programa Cardiovascular de un Centro de Salud Familiar (CESFAM) de Macul, Región Metropolitana, Chile, previo consentimiento informado. Muestreo aleatorio simple (confianza 95\%, merma 10\%). Recolección de información en CESFAM y/o visita domiciliaria por investigadoras e internas de enfermería entrenadas. Satisfacción del paciente, medida con subescala satisfacción del cuestionario Diabetes Quality of Life (EsDQOL). Análisis con estadística descriptiva, $\mathrm{Chi}^{2}$, Odd Ratios, IC (95\%). Resultados: Población mayoritariamente adulta mayor, femenina, promedio años estudio 8,7. El 42,6\% tiene diagnóstico de DM hace más de 10 años. Refieren mayor satisfacción con: tratamiento medicamentoso, controles recibidos, vida en general. Existe mayor insatisfacción con: tiempo ocupado en ejercicios, cómo duerme, vida sexual. Satisfacción con tratamiento medicamentoso se asocia a compensación de diabetes $(\mathrm{p}=0,026)$. Pacientes insatisfechos con tratamiento tienen 2 veces más riesgo de descompensación que los satisfechos, IC [1,08-3,7]. Insatisfacción con tiempo dedicado a enfermedad, controles, tiempo ocupado en controles y ejercicio indicado se asocian a complicaciones $(\mathrm{p}<0,05)$. Conclusión: Insatisfacción con características del tratamiento de DM2 se asocia mayormente a complicaciones que a compensación de DM, pacientes insatisfechos tienen más riesgo de complicaciones que los satisfechos.
\end{abstract}

Palabras clave: Diabetes mellitus tipo 2, satisfacción del paciente, atención de salud, enfermería en atención primaria.

\footnotetext{
${ }^{1}$ Financiamiento: Servicio de Salud Metropolitano Oriente (SSMO), Santiago, Chile y Fondo de Ayuda a la Investigación (FAI), Universidad de los Andes. Santiago, Chile.

*Enfermera. Docente Universidad de los Andes. Santiago, Chile. Email: magalianog@uandes.cl.

** Enfermera. Docente Universidad de los Andes. Santiago, Chile. Email: calsilvia@gmail.com.

${ }^{* * *}$ Enfermera. Docente Universidad de los Andes. Santiago, Chile. Email: mafeitotapia@gmail.com.

${ }^{* * * *}$ Enfermera. CESFAM Félix de Amesti. Santiago, Chile. Email: maria_aliaga@vtr.net.

${ }^{* * * * *}$ Enfermera. CESFAM Félix de Amesti. Santiago, Chile. Email: sleiva@corpomunimacul.cl.

${ }^{* * * * * *}$ Estadístico. Docente Universidad de los Andes. Santiago, Chile. Email: bmujica@vtr.net.
} 


\begin{abstract}
Diabetes Mellitus is increasing in our country, with a high prevalence of complications mainly because of poor metabolic control. Objectives: to evaluate association of health condition, demographic variables, and years since diagnosis with patient satisfaction regarding disease treatment. Methodology: prevalence study, 340 patient taken out of a population of 1.100 patients attended in the Cardiovascular Program of an outpatient clinic in Macul district. Informed consent was requested. Simple randomized sample (CI 95\%, loss 10\%). Data collected in the outpatient clinic or through home visits done by researchers or trained senior nursing students. Patient satisfaction was measured by the satisfaction subscale of the Diabetes Quality of Life Questionnaire (EsDQOL). Analysis: descriptive statistics, $\mathrm{Chi}^{2}$, Odds Ratios, CI (95\%). Results: Population of mainly older adults, female, average years of school attendance 8.7. 42.6\% has been diagnosed since more than 10 years. They refer greater satisfaction with: pharmacologic treatment, care received in outpatient visits, life in general. There exists more dissatisfaction with: time spent in exercise, sleep, sexual life. Satisfaction with pharmacologic treatment has association with diabetes compensation $(\mathrm{p}=0.026)$. Patients dissatisfied with treatment have 2 times more risk of poor metabolic control than satisfied patients, IC[1.08-3.7]. Dissatisfaction with time spent in the disease, outpatient visits, and time spent in exercises, are associated with complications ( $\mathrm{p}<$ 0.05). Conclusion: Dissatisfaction is more associated to complications than to poor metabolic control. Patient dissatisfied have more risk of complications than those satisfied.
\end{abstract}

Key words: Type 2 diabetes mellitus, patient satisfaction, health care, primary care nursing.

Fecha recepción: 29/11/11 Fecha aceptación: 29/09/12

\section{INTRODUCCIÓN}

La diabetes mellitus (DM) va en aumento en nuestro país. La prevalencia en adultos de esta enfermedad aumentó de $6.3 \%$ el año 2003 a 9,4\% el 2010 (1), observándose una alta prevalencia de complicaciones especialmente por un mal control metabólico (2) acentuado por la dificultad de seguir un tratamiento farmacológico y no farmacológico de por vida $(1,3)$.

La diabetes produce un deterioro gradual y progresivo en diferentes áreas de la vida de las personas según el avance de la enfermedad $(4,5)$; las áreas más afectadas son la capacidad funcional y la sensación de bienestar. En suma, la enfermedad, su manejo y las complicaciones impactan en diferentes aspectos de la vida cotidiana de los pacientes diabéticos, como en el trabajo, las relaciones personales, sociales y familiares y el bienestar físico y psicológico, entre otros $(1,2)$. Las complicaciones, como la vasculopatía perifé- rica, la retinopatía, la cardiopatía isquémica y la nefropatía, entre otras, son las que tienen mayor impacto en la vida de las personas (2, 6). Un buen control metabólico reduce las complicaciones macrovasculares, pero más aún las complicaciones microvasculares (5, 7). Los grupos de diabéticos más afectados en muchos aspectos de la vida cotidiana son las mujeres, los obesos, los ancianos, las personas que viven solas y las personas con bajo ingreso económico $(8,9)$.

La falta de un manejo efectivo de estos pacientes, según el Ministerio de Salud (MINSAL) (10), y la baja adherencia a las acciones recomendadas, según otros autores $(10,11)$, quedan de manifiesto en la baja proporción de pacientes bien controlados; sólo el 36\% de los pacientes diabéticos en la Atención Primaria en Chile tiene buen control metabólico (10). Hay un creciente número de pacientes diabéticos que ingresa a diálisis, una alta frecuencia de pacientes con pie diabético que deben ser amputados y otras complicaciones prevenibles que afectan significativamen- 
te la calidad de vida de las personas (7). La diabetes es el principal factor de riesgo para amputaciones de extremidades inferiores y la primera causa de amputación no traumática; sin embargo, la tasa de amputación por complicaciones periféricas, en población diabética, aumentó en Chile entre los años 2002 y 2006 en un 28\% (12).

El Ministerio de Salud plantea que en la atención primaria ya no basta con medir parámetros clínicos, sino que es necesario valorar otros aspectos (10), esta recomendación la comparten otros autores $(13,14)$, quienes plantean que además es necesario tener en cuenta la satisfacción del paciente con su tratamiento, ya que ésta, está relacionada con la adherencia terapéutica $(13,15)$ y los resultados clínicos (14), es decir, la reducción de los síntomas y el control de la enfermedad. Las consecuencias de los tratamientos satisfactorios frente a los insatisfactorios es que pueden tener un impacto directo sobre la eficacia terapéutica, especialmente cuando el tratamiento necesita de un autocontrol importante durante el resto de la vida, como es el caso de la diabetes $(14,15)$. Por lo mismo, hoy se tiende hacia un modelo de decisiones sobre el control de la enfermedad compartidas, en que el paciente va cobrando protagonismo $(13,15)$. Es posible que el hecho de conocer el grado de satisfacción del paciente con su tratamiento pueda contribuir a predecir la adherencia a éste por parte del paciente y ayudar al profesional en la toma de decisiones (13).

La enfermera tiene un papel preponderante dentro del equipo de salud que atiende estos pacientes. Muchas de las actividades recomendadas por el MINSAL en el Programa Cardiovascular (10) las realiza la enfermera; una de las principales es la educación al paciente y su familia sobre la enfermedad; la importancia de la adherencia al tratamiento farmacológico y no farmacológico para controlar la diabetes y prevenir complicaciones, aspectos fundamentales para una mejor calidad de vida. La enfermera de atención primaria que trabaja con estos enfermos crónicos dedica muchas horas de su jornada laboral a estas actividades, las cuales debe ajustar a cada paciente en particular para hacerlo partícipe del control de su enfermedad.

Preguntas de investigación: ¿Cuál es el nivel de satisfacción del paciente diabético tipo 2, controlado en un Centro de Salud Familiar de Macul (CESFAM), con las características específicas del tratamiento para controlar su enfermedad? ¿Qué variables se asocian a la satisfacción del paciente con las características específicas del tratamiento de la diabetes? Objetivos: Caracterizar a la población según variables sociodemográficas, tiempo de diagnóstico y condición de salud. Medir satisfacción de los pacientes con las características específicas del tratamiento de la diabetes. Identificar variables asociadas a la satisfacción del paciente con las características específicas del tratamiento de la diabetes.

Variables principales: 1) Condición de salud medida a través de: compensación de la diabetes (HbA1c < a 7\%), patologías crónicas agregadas según diagnóstico médico (hipertensión arterial (HTA), dislipidemia, insuficiencia renal, entre otras), grado de autovalencia según última encuesta de detección de riesgo de pérdida de funcionalidad en el adulto mayor (EFAM). Complicaciones de la diabetes según diagnóstico médico (nefropatía diabética incipiente, insuficiencia renal crónica, neuropatía diabética, amputación, retinopatía diabética, ceguera, infarto agudo del miocardio, accidente cerebrovascular ACV). Riesgo de pie diabético categorizado en riesgo máximo, alto y moderado de acuerdo a Norma clínica Manejo Integral del Pie diabético (10). Estado nutricional según diagnóstico nutricional en último control. Presión arterial medida al momento del estudio.

2) Satisfacción del paciente con las características específicas del tratamiento de la diabetes: se mide a través de la subescala de satisfacción del cuestionario Diabetes Quality of Life (EsDQOL), la cual consta de 15 preguntas $(16,17)$. 


\section{MATERIAL Y MÉTODO}

Estudio cuantitativo de prevalencia puntual a abril de 2010 de 1.100 pacientes diabéticos tipo 2 controlados en el Programa Cardiovascular en un Centro de Atención Primaria del Servicio de Salud Metropolitano Oriente (SSMO). Criterios de inclusión: paciente diabético tipo 2 diagnosticado de acuerdo a criterio de Guía Clínica de Diabetes Mellitus tipo 2 del Ministerio de Salud 2006, de 20 años o más, con a lo menos 1 año en control. Criterios de exclusión: pacientes con diagnóstico de demencia registrado en ficha electrónica y pacientes en Programa de Postrados. Muestra de 340 pacientes, asignando a las estimaciones una confianza del $95 \%$ con un margen de error del 5\%, conociendo que el tamaño de la población es de 1.100 pacientes y que se puede tener una merma del 10\%. Selección de la muestra por aleatorización simple por programa computacional. Los pacientes incluidos aceptaron participar en el estudio a través de consentimiento informado.

Recolección de la información: fue realizada por las investigadoras y por internas de enfermería de la Universidad de los Andes, previamente entrenadas. Se citó a los pacientes al centro de salud; de no cumplir con la citación, se les realizó una visita domiciliaria. La información sociodemográfica del paciente se recolectó a través de una encuesta probada previamente y revisada por expertos. La información sobre la condición de salud se extrajo de la ficha electrónica, considerando los siguientes componentes: 1 . Compensación de la diabetes, objetivada por las cifras de hemoglobina glicosilada ( $\mathrm{Hb}$ Ac1 < a 7\%). 2. Patologías crónicas agregadas con confirmación diagnóstica, categorizadas en hipertensión arterial, dislipidemia, insuficiencia renal, otras. 3. Complicaciones de la diabetes según diagnóstico médico de nefropatía diabética incipiente, insuficiencia renal crónica, neuropatía diabética, amputación, retinopatía diabética, ceguera, infar- to agudo del miocardio y ACV. 4. Riesgo de pie diabético: categorizado en riesgo máximo, alto y moderado de acuerdo a "Norma Técnica Manejo Integral del Pie Diabético" (10). 5. Diagnóstico nutricional expresado en: normal, sobrepeso, obeso y enflaquecido.

La satisfacción del paciente con características específicas del tratamiento de la diabetes se mide a través de la subescala de satisfacción del cuestionario Diabetes Quality of Life (EsDQOL), la cual consta de 15 preguntas. Las respuestas se cuantifican usando una escala de Likert de 5 respuestas ordinales con un rango que va desde 1 , correspondiente a muy satisfecho, a 5, correspondiente a nada satisfecho. Si el paciente tiene una puntuación global de 15 , se trata de un paciente muy satisfecho. Esta variable también se mide considerando las respuestas "muy satisfecho" y "bastante satisfecho" como una categoría (satisfecho) y "algo", "poco" o "nada satisfecho" como otra categoría (insatisfecho).

La técnica de análisis de los datos y la evaluación de los resultados se realizó a través de los programas computacionales Stata 5.0, Epi Info 3.5.1 y Excel 2003. Se utilizaron estadística descriptiva y medidas de resumen para caracterizar al paciente. Para la asociación de variables se utilizó Chi y para la comparación de medias se utilizó t de Student; se determinaron Odd Ratios con sus intervalos correspondientes con un $95 \%$ de confianza.

El proyecto de investigación fue aprobado por el Comité de Ética del SSMO y el Comité de Ética de la Universidad de los Andes.

\section{RESULTADOS}

La población estudiada se caracteriza en la Tabla 1. El promedio de edad es de 66,5 años (DS 10,8), predominando el sexo femenino. La escolaridad promedio es de 8,7 años. Las mujeres tienen menor nivel de escolaridad que los hombres, $\mathrm{X}^{2}=25.8845 ; \mathrm{p}=0,000$ (Tabla 2), el 47,8\% de ellas tiene menos de 
Tabla 1. Caracterización de los pacientes estudiados $(\mathrm{n}=340)$.

\begin{tabular}{|c|c|c|}
\hline Características & $\mathbf{n}$ & $\%$ \\
\hline \multicolumn{3}{|l|}{ Edad } \\
\hline$<65$ años & 141 & 41,5 \\
\hline 65 años y más & 199 & 58,5 \\
\hline$\overline{\mathrm{x}}$ edad (DS) & 66.5 & 10.8 \\
\hline \multicolumn{3}{|l|}{ Sexo } \\
\hline Femenino & 208 & 61,2 \\
\hline Masculino & 132 & 38,8 \\
\hline \multicolumn{3}{|l|}{ Nivel educacional } \\
\hline $\bar{x}$ años de estudio (DS) & 8,7 & 4,4 \\
\hline Sin estudio & 19 & 5,6 \\
\hline Básica incompleta & 115 & 33,8 \\
\hline Básica completa & 30 & 8,8 \\
\hline Media incompleta & 59 & 17,4 \\
\hline Media completa & 76 & 22,4 \\
\hline Estudio superior & 40 & 11,8 \\
\hline Sin registro & 1 & 0,3 \\
\hline \multicolumn{3}{|l|}{ Tipo de Familia } \\
\hline Nuclear & 164 & 48,2 \\
\hline Extendida & 148 & 43,5 \\
\hline Vive solo(a) & 28 & 8,2 \\
\hline \multicolumn{3}{|l|}{ Ingreso familiar } \\
\hline Menor a $\$ 150.000$ & 152 & 44,7 \\
\hline$\$ 150.000$ a $\$ 300.000$ & 132 & 38,7 \\
\hline$>\$ 300.000$ & 56 & 16,6 \\
\hline \multicolumn{3}{|l|}{ Actividad laboral } \\
\hline Estudiante & 1 & 0,29 \\
\hline Dueña(o) de casa & 143 & 42,06 \\
\hline Sin actividad por Jubilación o pensión & 93 & 27,35 \\
\hline Cesante & 4 & 1,18 \\
\hline Trabajador & 99 & 29,12 \\
\hline \multicolumn{3}{|l|}{ Tiempo de diagnóstico de la diabetes } \\
\hline$\leq \mathrm{a} 10$ años & 195 & 57,4 \\
\hline$>$ a 10 años & 145 & 42,6 \\
\hline
\end{tabular}

Tabla 2. Nivel educacional según sexo.

\begin{tabular}{lrrrr}
\hline & \multicolumn{2}{c}{ Hombres } & Mujeres \\
\hline Nivel educacional & $\mathrm{N}$ & $\%$ & $\mathrm{~N}$ & $\%$ \\
\hline Sin estudio & 3 & 2,3 & 16 & 7,7 \\
Básica incompleta & 32 & 24,2 & 83 & 39,9 \\
Básica completa & 12 & 9,1 & 18 & 8,7 \\
Media incompleta & 21 & 15,9 & 38 & 18,3 \\
Media completa & 38 & 28,8 & 38 & 18,3 \\
Estudio superior & 26 & 19,7 & 14 & 6,7 \\
Sin registro & 0 & 0 & 1 & 0,5 \\
\hline Total & 132 & 100 & 208 & 100 \\
\hline
\end{tabular}

$\mathrm{Chi}^{2}=25,8845 ; \mathrm{p}=0,000$ 
8 años de estudio a diferencia de los varones $(26,5 \%)$. El $42 \%$ de los pacientes desempeña labores de dueña(o) de casa. Un 42,6\% tiene diagnosticada la enfermedad hace más de 10 años.

$\mathrm{Al}$ analizar la condición de salud (Tabla 3 ), se observa que el $43,5 \%$ de los pacientes está descompensado de la diabetes ( $\mathrm{HbAcl}$ $\geq 7 \%$ ), sin diferencia estadísticamente significativa según sexo. El 97,9\% presenta alguna patología agregada; de ellas, las más frecuentes son la hipertensión arterial (HTA) y la dislipidemia, sin diferencia estadísticamente significativa según sexo. El $25 \%$ presenta alguna complicación de la diabetes; las más frecuentes son la retinopatía diabética $(41,2 \%)$, el infarto agudo del miocardio $(32,9 \%)$ y la insuficiencia renal crónica $(17,6 \%)$; no hay diferencia estadísticamente significativa según sexo. El 12,9\% de los pacientes presenta una amputación como complicación de la diabetes; los hombres tienen 7.5 veces más riesgo de amputación que las mujeres ( $\mathrm{p}=0,004$; IC 1,49-51,37). El $71,2 \%$ de los pacientes está con sobrepeso u obesidad. Las mujeres tienen 2.03 veces más riesgo de alteración nutricional por exceso que los hombres ( $\mathrm{p}=0,009$; IC 1,23 $3,37)$. El 23,1\% de los adultos mayores (199) es autovalente.

Tabla 3. Condición de salud de los pacientes según sexo.

\begin{tabular}{|c|c|c|c|c|c|c|c|c|c|}
\hline \multirow[b]{2}{*}{ Condición de salud $\quad(n=340)$} & \multicolumn{4}{|c|}{ Hombres } & \multicolumn{2}{|c|}{ Mujeres } & \multirow[b]{2}{*}{$p$} & \multirow[b]{2}{*}{$\mathrm{OR}$} & \multirow[b]{2}{*}{ IC } \\
\hline & Total & $\%$ & $\mathrm{~N}^{\circ}$ & $\%$ & $\mathrm{~N}^{\circ}$ & $\%$ & & & \\
\hline Descompensación de la diabetes & 148 & 43,5 & 60 & 40,5 & 88 & 59,5 & 0,568 & & \\
\hline Patología Agregada (1 o más) & 333 & 97,9 & 129 & 38,7 & 204 & 61,3 & 0,555 & & \\
\hline - HTA & 310 & 93,09 & 119 & 38,4 & 191 & 61,6 & 0,595 & & \\
\hline - Dislipidemia & 221 & 66,4 & 90 & 40,7 & 131 & 59,3 & 0,327 & & \\
\hline - Insuficiencia renal & 16 & 4,8 & 10 & 62,5 & 6 & 37,5 & 0,046 & & \\
\hline - Otras & 134 & 40,2 & 49 & 36,6 & 85 & 63,4 & 0,491 & & \\
\hline Complicaciones de la diabetes (1 o más) & 85 & 25 & 39 & 45,9 & 46 & 54,1 & 0,123 & & \\
\hline - Nefropatía diabética incipiente & 10 & 11,8 & 6 & 60 & 4 & 40 & 0,195 & & \\
\hline - Insuficiencia renal crónica & 15 & 17,6 & 9 & 60 & 6 & 40 & 0,085 & & \\
\hline - Neuropatía diabética & 11 & 12,9 & 5 & 45,5 & 6 & 54,5 & 0,646 & & \\
\hline - Amputación & 11 & 12,9 & 9 & 81,8 & 2 & 18,2 & 0,004 & 7,54 & $1,49-51,37$ \\
\hline - Retinopatía diabética & 35 & 41,18 & 18 & 51,4 & 17 & 48,6 & 0,106 & & \\
\hline - Ceguera & 5 & 5,9 & 1 & 20 & 4 & 80 & 0,356 & & \\
\hline - Infarto agudo del miocardio & 28 & 32,9 & 14 & 50 & 14 & 50 & 0,205 & & \\
\hline - Accidente vascular encefálico & 12 & 14,12 & 5 & 41,7 & 7 & 58,3 & 0,529 & & \\
\hline Riesgo de pie diabético alto, máximo & 133 & 39,12 & 57 & 42,9 & 76 & 57,1 & 0,221 & & \\
\hline Descompensación presión arterial & 198 & 58,2 & 84 & 42,4 & 114 & 57,6 & 0.642 & & \\
\hline Estado nutricional alterado por exceso & 242 & 71,2 & 82 & 33,9 & 160 & 66,1 & 0,009 & 2,03 & $1,23-3,37$ \\
\hline Autovalencia adultos mayores $(\mathrm{n}=199)$ & 46 & 23,1 & 18 & 39,1 & 28 & 60,9 & 0,944 & & \\
\hline
\end{tabular}

La puntuación promedio de la variable satisfacción con las características específicas del tratamiento de la diabetes fue 31 (DS.8.42). Al analizar los ítemes de esta variable en forma dicotómica ("satisfecho", "no satisfecho") según se observa en Tabla 4, el $85,9 \%$ está satisfecho con el tratamiento medicamentoso, el $83,3 \%$ con los controles reci- bidos y un $83,2 \%$ con la vida en general; sólo el $34,4 \%$ está satisfecho con su vida sexual y un $54,7 \%$ está satisfecho con cómo duerme. Un $55,6 \%$ está satisfecho con el tiempo que ocupa en ejercicios; los pacientes de 65 años y más están más satisfechos con el tiempo que le dedican al ejercicio $(63,8 \%)$ que los menores de esa edad (43,9\%), $X^{2}=13,17, p=0,000$. 
Tabla 4. Satisfacción del paciente con las características específicas de tratamiento de la diabetes y asociación con compensación y con complicaciones de la diabetes $(\mathrm{n}=340)$.

\begin{tabular}{|c|c|c|c|c|c|c|}
\hline \multirow[b]{2}{*}{$\begin{array}{l}\text { Itemes de subescala de satisfacción del } \\
\text { cuestionario EsDQOL }\end{array}$} & \multicolumn{2}{|c|}{ Satisfechos } & \multicolumn{2}{|c|}{ No satisfechos } & \multirow{2}{*}{$\begin{array}{c}\text { Asociación entre } \\
\text { c/ítem de subescala } \\
\text { de satisfacción y } \\
\text { compensación de la } \\
\text { diabetes }\end{array}$} & \multirow{2}{*}{$\begin{array}{c}\text { Asociación entre } \\
\text { c/ítem de subescala } \\
\text { de satisfacción y } \\
\text { complicaciones de la } \\
\text { diabetes } \\
\text { p }\end{array}$} \\
\hline & $\mathrm{n}$ & $\%$ & $\mathrm{n}$ & $\%$ & & \\
\hline Tiempo que le dedica a la enfermedad & 261 & 76,8 & 79 & 23,2 & 0,087 & 0,022 \\
\hline Controles & 283 & 83,3 & 57 & 16,7 & 0,522 & 0,024 \\
\hline Tiepo ocupado en controles & 263 & 77,4 & 77 & 22,6 & 0,511 & 0,043 \\
\hline Tratamiento medicamentoso & 292 & 85,9 & 48 & 14,1 & 0,026 & 1,00 \\
\hline Dieta indicada & 253 & 74,4 & 87 & 25,6 & 0,974 & 0,830 \\
\hline Ejercicio indicado & 205 & 60,2 & 135 & 39,8 & 0,537 & 0,035 \\
\hline Apoyo familiar a su enfermedad & 267 & 78,6 & 73 & 21,4 & 0,460 & 0,147 \\
\hline Como duerme & 186 & 54,7 & 154 & 45,3 & 0,275 & 0,706 \\
\hline Relaciones sociales & 264 & 77,6 & 76 & 22,4 & 0,418 & 0,764 \\
\hline Vida sexual & 117 & 34,4 & 223 & 65,6 & 0,256 & 0,015 \\
\hline Actividades que realiza de la vida diaria & 273 & 80,3 & 67 & 19,7 & 0,818 & 0,479 \\
\hline Tiempo que ocupa en ejercicios & 189 & 55,6 & 151 & 44,4 & 0,617 & 0,051 \\
\hline Tiempo libre & 268 & 78,8 & 72 & 21,2 & 0,720 & 0,125 \\
\hline Vida en general & 283 & 83,2 & 57 & 16,8 & 0,220 & 0,675 \\
\hline
\end{tabular}

La compensación de la diabetes se asocia a la satisfacción con tratamiento medicamentoso, $\mathrm{X}^{2}=4,98, \mathrm{p}=0,026$. Los pacientes insatisfechos con el tratamiento tienen 2 veces más riesgo de descompensación que los satisfechos IC [1,08-3,7]. Las complicaciones se asocian a insatisfacción con: el tiempo que le dedica a la enfermedad $\left(\mathrm{X}^{2}=5,28, \mathrm{p}=\right.$ $0,022)$, los controles $\left(\mathrm{X}^{2}=5,12, \mathrm{p}=0,024\right)$, el tiempo ocupado en controles $\left(\mathrm{X}^{2}=4,08, \mathrm{p}=\right.$ $0,043)$ la vida sexual $\left(X^{2}=5,95, p=0,015\right)$ y los ejercicios $\left(\mathrm{X}^{2}=4,46, \mathrm{p}=0,035\right)$.

\section{DISCUSIÓN Y CONCLUSIÓN}

La población estudiada tiene características similares a las de otros estudios $(18,19)$; predominan las mujeres, los adultos mayores y las personas de baja escolaridad; en estudios previos $(18,19)$ el nivel de escolaridad de la población es aún más bajo. La proporción de pacientes que padece la enfermedad hace más de 10 años (42,6 \%) es mayor a lo que refieren otros autores $(20,21)$ en sus estudios $(22,2 \%$ y $31,4 \%$ respectivamente $)$, lo que pudiera deberse a que en nuestro país se diagnostica más tempranamente la enfermedad.

Si bien un 56,5\% de los pacientes diabéticos está compensado, proporción mayor a lo encontrado en la Encuesta Nacional de Salud 2010 (34,32\%) (1), y en otros estudios internacionales $(30 \%$ y menos) $(18,19)$, sigue existiendo un $43,5 \%$ de pacientes con un mal control metabólico, lo que se traduce en un mayor riesgo de complicaciones y, por lo tanto, peores condiciones de vida (1, $2,6)$. Esta proporción de pacientes con mal control metabólico debiera ser una prioridad 
para los profesionales de la salud, especialmente para las enfermeras, ya que la educación a los pacientes, especialmente en relación a la adherencia al tratamiento, el apoyo y seguimiento es fundamental para prevenir las complicaciones $(10,22)$.

La alta frecuencia de HTA encontrada en los pacientes se observa también en otros estudios $(9,20,21)$, al igual que la alteración nutricional por exceso y la dislipidemia. La literatura refiere que las enfermedades asociadas a la diabetes y las complicaciones deterioran en forma significativa la capacidad funcional y la sensación de bienestar de las personas (3) y, a medida que los pacientes diabéticos presentan sobrepeso u obesidad, se afecta directamente su capacidad física y su bienestar psicológico (1). En este estudio las complicaciones se asocian a insatisfacción con las atenciones brindadas por la institución de salud, lo que de alguna manera avala lo expuesto por el MINSAL y otros estudios $(10,11)$ sobre la falta de un manejo efectivo de estos pacientes en las instituciones de salud y la baja adherencia a las acciones recomendadas $(13,14,15)$.

Un 25\% de los pacientes estudiados tienen complicaciones de la diabetes, coincidiendo las más frecuentes (retinopatía diabética, infarto agudo del miocardio e insuficiencia renal crónica) con lo expuesto por otros autores $(2,8)$. Es preocupante la tasa de amputación encontrada (12,9\%) en este estudio, más aún si se considera que es una complicación prevenible. Al igual que a nivel nacional, los hombres tienen mayor riesgo de amputación que las mujeres. Uno de los factores que podría contribuir a esto es que los hombres tienen una menor conciencia de autocuidado, reflejado en la menor asistencia a los centros de atención de salud que las mujeres. Aquí nuevamente la labor de la enfermera es fundamental para revertir o disminuir este problema.

Los estudios que miden la satisfacción del paciente diabético con su tratamiento, utilizan una gran diversidad de instrumentos, lo que dificulta la comparación de los resultados.

Los resultados obtenidos en este estudio reflejan una proporción importante de pacientes satisfechos con la vida en general $(83,2 \%)$. Diversos autores $(19,23,24)$ plantean que los hombres tienen mayor satisfacción con el tratamiento que las mujeres; en nuestro estudio no se observa esta diferencia. De los tres principales aspectos del tratamiento percibidos con mayor satisfacción, dos corresponden a atenciones entregadas por la institución de salud, a diferencia de los aspectos peor percibidos que corresponden a situaciones de la vida diaria. La asociación encontrada entre compensación de la diabetes y satisfacción con el tratamiento coincide con lo encontrado en el estudio de Cárdenas (19).

En conclusión, la insatisfacción del paciente diabético con características específicas del tratamiento para su enfermedad se asocia mayormente a complicaciones que a compensación de la DM, pacientes insatisfechos tienen más riesgo de complicaciones que los satisfechos. Las características específicas del tratamiento correspondientes a la vida diaria están dadas principalmente por la insatisfacción con la vida sexual, con cómo duerme y con el tiempo que ocupa en ejercicios.

Los pacientes con complicaciones de la diabetes refieren menor satisfacción que los pacientes descompensados de la enfermedad.

\section{REFERENCIAS}

1. Ministerio de Salud, Departamento de Epidemiología. Encuesta Nacional de Salud [Internet]. Chile: Ministerio de Salud; 2009-2010. [citado 10 abril 2010]. Disponible en: http://epi.minsal.cl/

2. Zafra JA, Méndez JC, Novalbos JP. Costa MJ, Fraílde I. Complicaciones crónicas 
en los pacientes con diabetes mellitus tipo 2 atendidos en un centro de salud. Aten Primaria. 2000; 25(8): 29-43.

3. Mena FJ, Martín JC, Simal F, Bellido J, Carretero JL. Diabetes Mellitus tipo 2 y calidad de vida relacionada con la salud: resultados del Estudio Hortega. An Med Interna. 2006; 23(8): 357-60.

4. Hervás A, Zabaleta A, De Miguel G, Beldarrain O, Diez J. Calidad de vida relacionada con la salud en pacientes con diabetes mellitus tipo 2. Anales Sis San Navarra. 2007; 30(1): 45-52.

5. De los Ríos JL, Sánchez JJ, Barrios P, Avila TL. Quality of life in patients with diabetic nephropathy. Invest Educ Enferm. 2005; 23(1): 30-43.

6. Vermeire E, Wens J, Van Royen P, Biot $\mathrm{Y}$, Hearnshaw $\mathrm{H}$, Lindenmeyer A. Intervenciones para mejorar el cumplimiento de las recomendaciones de tratamiento en personas con diabetes mellitus tipo 2 (Revisión Cochrane traducida). En: La Biblioteca Cochrane Plus [Internet]. Oxford: Update Software Ltd. 2008 [citado 17 agosto 2010]. Disponible en: http:// www.update-software.com. Traducida de The Cochrane Library 2008 Issue 3. Chichester, UK: John Wiley \& Sons Ltd.

7. Bradley C, De Pablos-Velasco P, Parhoferc KG, Eschwèged E, Gönder-Frederick L, Simonf D. PANORAMA: A European study to evaluate quality of life and treatment satisfaction in patients with type-2 diabetes mellitus-Study design. Prim Care Diabetes. 2011; 5(4): 231-9.

8. Quirantes A, López L, Curbelo V, Montano JA, Machado P, Quirantes A. La calidad de la vida del paciente diabético. Rev Cubana Med Gen Integr [Internet]. 2000 [citado 13 abril 2010]; 16(1): 50-6 Disponible en: http://scielo.sld.cu/pdf/mgi/ v16n1/mgi09100.pdf

9. Deakin T, McShane CE, Cade JE, Williams RDRR. Entrenamiento grupal de estrategias de autocuidado en personas con diabetes mellitus tipo 2 (Revisión
Cochrane traducida). En: La Biblioteca Cochrane Plus [Internet]. Oxford: Update Software Ltd. 2008 [citado 4 mayo 2010]. Disponible en: http://www.update-software.com. Traducida de The Cochrane Library 2008 Issue 3. Chichester, UK: John Wiley \& Sons Ltd.

10. Ministerio de Salud. Guía Clínica Diabetes Mellitus tipo 2 [Internet]. Santiago, Chile: MINSAL; 2009 [citado 13 abril 2010]. Disponible en: http://www. redcronicas.cl/index.php?option $=\mathrm{com}_{-}$ docman\&Itemid $=114$

11. McGlynn EA, Asch SM, Adams J, Keesey J, Hicks J, DeCristoforo A et al. The Quality of Health Care Delivered to Adults in the United States. N Engl J Med. 2003; 348: 2635-645.

12. Ministerio de Salud. Estrategia Nacional de Salud para el cumplimiento de los Objetivos Sanitarios de la Década 2011-2020 [Internet]. Santiago, Chile: MINSAL; 2011 [citado 13 abril 2012] Disponible en:http://www.minsal.gob.cl/ portal/docs/1/5648346.pdf

13. Villar J, Lizán L, Soto J, Peiró S. La satisfacción con el tratamiento. Aten Primaria. 2009; 41(11): 637-645.

14. Testa M. Mejorar la terapia de diabetes: aumentar la satisfacción. Diabetes Voice 2003; 48(4): 23-25.

15. Badia X. La satisfacción con el tratamiento. Med Clin (Barc). 2005; 125(3): 98-9.

16. Millán M. Cuestionario de calidad de vida específico para la diabetes mellitus (ESDQOL). Aten Primaria. 2002; 29(8): 517-21.

17. Millán M, Reviriego J, Del Campo J. Revaluación de la versión española del cuestionario Diabetes Quality of Life (EsDQOL). Endocrinol Nutr. 2002; 49(10): 322-4.

18. López-Carmona JM, Rodríguez-Moctezuma R. Adaptación y validación del instrumento de calidad de vida Diabetes 39 en pacientes mexicanos con diabetes mellitus tipo 2. Salud pública Méx. 2006; 
48(3): 200-11.

19. Cárdenas V, Pedraza C, Lerma R. Calidad de vida del paciente con diabetes mellitus tipo 2. Ciencia UANL. 2005; 8(3): 351-57.

20. Vicente B, Zerquera G, Peraza D, Castañeda E, Irizar J, Valladares T. Calidad de vida en el paciente diabético. MediSur [Internet]. 2008 [citado 15 marzo 2010]; 6(3): 22-8. Disponible en: http://www. medisur.sld.cu/index.php/medisur/issue/view/30

21. Vijan S, Hofer TP, Hayward RA. Cost-utility analysis of screening intervals for diabetic retinopathy in patients with type 2 diabetes mellitus. JAMA [Internet] 2000 [citado 4 abril 2010]; 283(7): 889-896. Disponible en: http://jama.ama-assn. org/content/283/7/889.full.pdf+html

22. Duke SAS, Colagiuri S, Coliaguri R. Educación individual del paciente con dia- betes mellitus tipo 2 (Revisión Cochrane traducida). En: La Biblioteca Cochrane Plus [Internet]. Oxford: Update Software Ltd. 2009 [citado 17 abril 2010]. Disponible en: http://www.update-software. com. Traducida de The Cochrane Library 2009 Issue 1. Chichester, UK: John Wiley \& Sons Ltd.

23. Árcega-Domínguez A, Lara-Muñoz C, Ponce-de-León-Rosales S. Factores relacionados con la percepción subjetiva de la calidad de vida de pacientes con diabetes. Rev Invest clin. 2005; 57(5): 676-84.

24. Mata M, Roset M, Badia X, Antoñanzas F, Ragel J. Impacto de la diabetes mellitus tipo 2 en la calidad de vida de los pacientes tratados en las consultas de atención primaria en España. Aten Primaria. 2003; 31(8): 493-9. 